Case Report

\title{
A retainer-free obturator prosthesis in a fully dentulous patient with palatal defects
}

\author{
Mamoru Murakami ${ }^{1)}$, Yasuhiro Nishi ${ }^{2)}$, Takaharu Shimizu ${ }^{2,3)}$, and Masahiro Nishimura ${ }^{2)}$ \\ 1) Denture Prosthodontics Restoration, Advanced Dentistry Center, Kagoshima University Hospital, Kagoshima, Japan \\ ${ }^{2)}$ Department of Oral and Maxillofacial Prosthodontics, Kagoshima University Graduate School of Medical and Dental Sciences, Kagoshima, Japan \\ 3) Private Practice, Kobe, Japan
}

(Received August 14, 2018; Accepted June 13, 2019)

\begin{abstract}
Retainers are the most important component in the success of an obturator prosthesis. However, the optimal retainer design for a fully dentulous patient with palatal defect needs four widely separated retainers - resulting in unsatisfactory esthetics - and regulates engagement of deep undercuts of the defect cavity, which leads to an inadequate palatal seal. A new retainer-free obturator prosthesis that closes only the defect cavity and is retained by undercuts within the defect was designed and proved useful for problems caused by a clasp-retained obturator prosthesis for a fully dentulous patient with palatal defects.
\end{abstract}

Keywords; maxillectomy, obturator, sectional denture, soft relining material

\section{Introduction}

Prosthetic obturation can improve mastication, speech, swallowing, and esthetics in maxillectomy patients [1]. In addition, a stable obturator prosthesis improves patient satisfaction [2]. Retainers are the most important component in the success of an obturator prosthesis [3]. To resist the multiple forces acting on an obturator prosthesis, a classification for maxillectomy patients [4] and an ideal retainer design for each class [3] have been proposed. For fully dentulous patients with defects in the center of the palate (Aramany class 3 defect), four widely separated retainers should be designed by using quadrilateral configurations [3].

Engagement of the undercuts of a defect cavity, including the anterior nasal aperture, lateral scar band, residual hard palate, and residual soft palate, can provide retention and a sufficient posterior palatal seal for an obturator prosthesis [1]. However, the configuration of four widely separated retainers regulates engagement of deep undercuts of the defect cavity, thus leading to an inadequate palatal seal. Additionally, multiple retainers affect factors such as esthetics, caries susceptibility, gingival health, and accumulation of food residue (Osborn J, Lammie GA. Partial dentures. 4th ed. Oxford: Blackwell scientific publications, 166-255, 1981).

Because occlusal force is not directly applied to the obturator prosthesis in such cases, utilization of the undercut of the defect cavity is preferable to placing the retainer so as to obtain effective obturation and acceptable esthetics. Therefore, a retainer-free obturator prosthesis was designed, and its effects on oral function and esthetics in a fully dentulous patient with defects of the hard and soft palate were assessed.

\section{Case Report}

In October 2013, a 57-year-old woman visited the Otolaryngology Department at Kagoshima University Hospital for evaluation of nasal congestion. Myoepithelioma of the palate was diagnosed, and maxillary bone and palate resection was planned. Later, the patient visited the Department of Denture Prosthodontics Restoration, in November 2013, to plan for postoperative prosthodontic treatment. Figure 1 shows intraoral views and

Correspondence to Dr. Mamoru Murakami, Denture Prosthodontics Restoration, Advanced Dentistry Center, Kagoshima University Hospital, 8-35-1 Sakuragaoka, Kagoshima 890-8544, Japan

Fax:+81-99-275-6228 E-mail: kaku@dent.kagoshima-u.ac.jp

Color figures can be viewed in the online issue at J-STAGE.

doi.org/10.2334/josnusd.18-0276

DN/JST.JSTAGE/josnusd/18-0276 magnetic resonance imaging (MRI) scans obtained during the patient's first visit. There were no missing teeth (Fig. 1a-c). MRI revealed a wellcircumscribed mass (diameter, $\approx 30 \mathrm{~mm}$ ) in the nasal cavity in the upper hard palate (Fig. 1d).

Palatal resection was conducted on November 13, 2013. Her chief complaints were nasal regurgitation and difficulty chewing. Intraoral examination revealed that no alveolar bone or teeth had been removed. However, the left inferior turbinate had been surgically resected, resulting in a large elliptical palatal defect extending from the hard palate to the soft palate (Fig. 2a), which corresponded to an Aramany class 3 defect [4] measuring $4 \times 3 \mathrm{~cm}$. She had received no radiation therapy or chemotherapy. Dysphagia and masticatory dysfunction were diagnosed. A clasp-retained obturator prosthesis was inserted 1 week after the surgery (Fig. 2a), with subsequent adjustments of the obturator around the defect cavity (Fig. 2b). Despite many adjustments, after the wound had healed the patient complained, in April 2014, of poor esthetics, accumulation of food residue around the retainer, and nasal drainage (Fig. 2b, c).

Table 1 shows the results, with and without the clasp-retained obturator prosthesis (April 2014), for masticatory function assessed with gummy jelly [2], velopharyngeal function, as indicated by blowing ratio [5], and subjective evaluations using a visual analog scale and mood assessment testing [2]. The results highlighted the following three problems. (1) The posterior palatal seal was insufficient because of the vertical path of insertion/removal of multiple retainers. Despite the flexibility of soft tissue and the varying rotation paths of the several obturator insertions attempted, extension onto the nasopharyngeal side of the soft palate was insufficient. (2) Poor esthetics of the circumferential clasp. (3) Food residue accumulation around the multiple retainers. It was decided that these problems could not be solved by fabricating a conventional clasp-retained obturator prosthesis, and the patient declined an obturator prosthesis with clasps. Hence, a retainer-free obturator prosthesis that enabled a large rotation path toward the undercut on the soft palate side was designed. Written informed consent was obtained from the patient for the fabrication of a retainer-free obturator prosthesis and the publication of this case report.

A final impression was obtained by using irreversible hydrocolloid (Algiace Z; Dentsply Sirona K.K, Tokyo, Japan), and a working model was fabricated. It was impossible to reproduce the large undercuts present in the defect cavity. Therefore, as shown in Fig. 3a, a retainer-free obturator prosthesis without undercuts was fabricated from this model by using cold-cured resin with a compressed air injection unit (Palajet System; Heraeus-Kulzer, Tokyo, Japan). After curing, the palatal plate and obturator were joined by five magnetic attachments (Gigauss D800 and D400; GC Corp., Tokyo, Japan). The keeper of each magnetic attachment was bonded to the top of the obturator with a surveyor and 4-META/MMATBB resin (Super-Bond, Clear; Sun Medical, Moriyama, Japan) at the dental laboratory. The amount and shape of the extension of the obturator was determined chairside by using self-curing resin (Unifast Trad; GC Corp.) and tissue conditioner (Tissue Conditioner 2; Shofu Inc., Kyoto, Japan) while checking the extent of obturation (Fig. 3b). The obturator portion extends upward along the outer edge around the defect and is not in contact with the nasal septum. To securely close the soft palate defect during function, the section of the obturator in the soft palate was extended so that the posterior border of the obturator contacted the oral side of the soft palate at rest (Fig. 3b; dashed arrow). In addition, the extended part of the obturator in the nasal cavity contacted the nasal side of the soft palate during elevation of the soft palate [1], (Fig. 3b, arrow).

In June 2014, after confirming that the patient's functional character- 


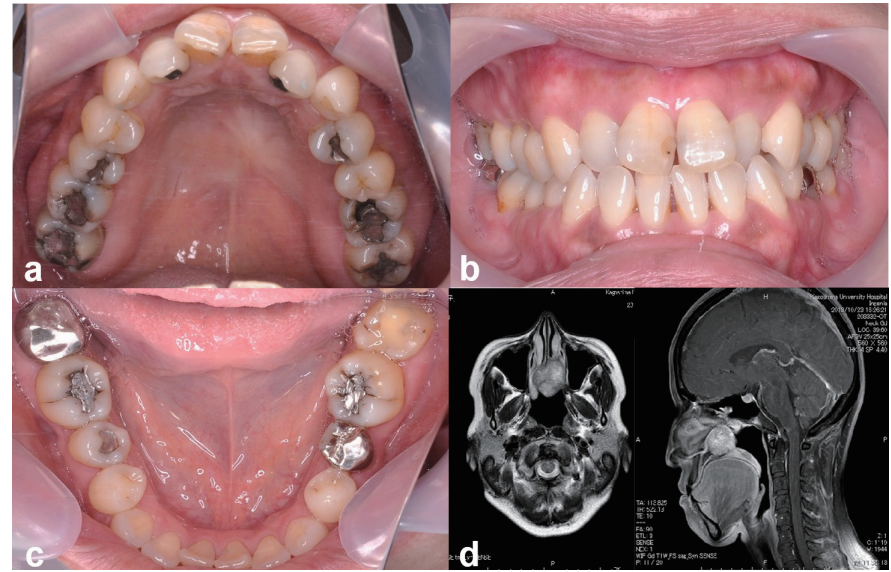

Fig. 1 Intraoral views (a, b, c) and magnetic resonance imaging (d) before surgical resection

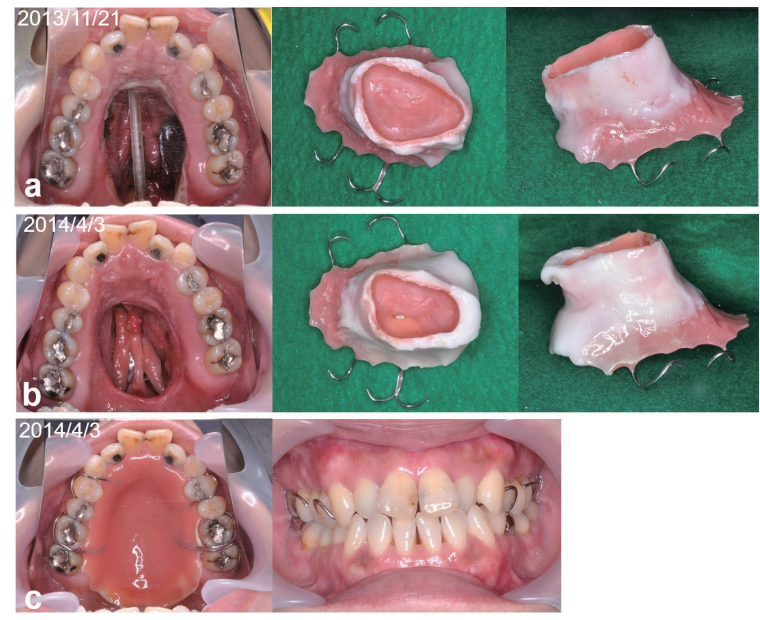

Fig. 2 Intraoral views and the clasp-retained obturator prosthesis on day 8 after surgery (a) and at 5 months after surgery (b). Intraoral views with the clasp-retained obturator prosthesis at 5 months after surgery (c)

Table 1 Patient results, with and without the obturator prosthesis in place

\begin{tabular}{|c|c|c|c|c|}
\hline & $\begin{array}{l}\text { Without obturator } \\
\text { (April 2014) }\end{array}$ & $\begin{array}{c}\text { Clasp-retained obturator } \\
\text { (April 2014) }\end{array}$ & $\begin{array}{l}\text { Retainer-free obturator } \\
\text { (September 2014) }\end{array}$ & $\begin{array}{l}\text { Retainer-free obturator } \\
\text { (June 2019) }\end{array}$ \\
\hline Glucose concentration after chewing gummy jelly $(\mathrm{mg} / \mathrm{dL})$ & 140.2 & 247.0 & 269.8 & 272.0 \\
\hline Blowing ratio $(\%)$ & 0 & 99.1 & 99.6 & 99.7 \\
\hline \multicolumn{5}{|l|}{ Visual analog scale $(100 \mathrm{~mm})$} \\
\hline Ability to swallow & & 66.6 & 97.0 & 100.0 \\
\hline Ability to chew & not measured & 53.9 & 100.0 & 100.0 \\
\hline Esthetics & & 54.6 & 99.2 & 100.0 \\
\hline Ease in cleaning & & 90.7 & 97.8 & 100.0 \\
\hline Mood of patient ( $1-7 ; 7$ is the worst, 1 is the best) & 7 & 5 & 2 & 2 \\
\hline
\end{tabular}
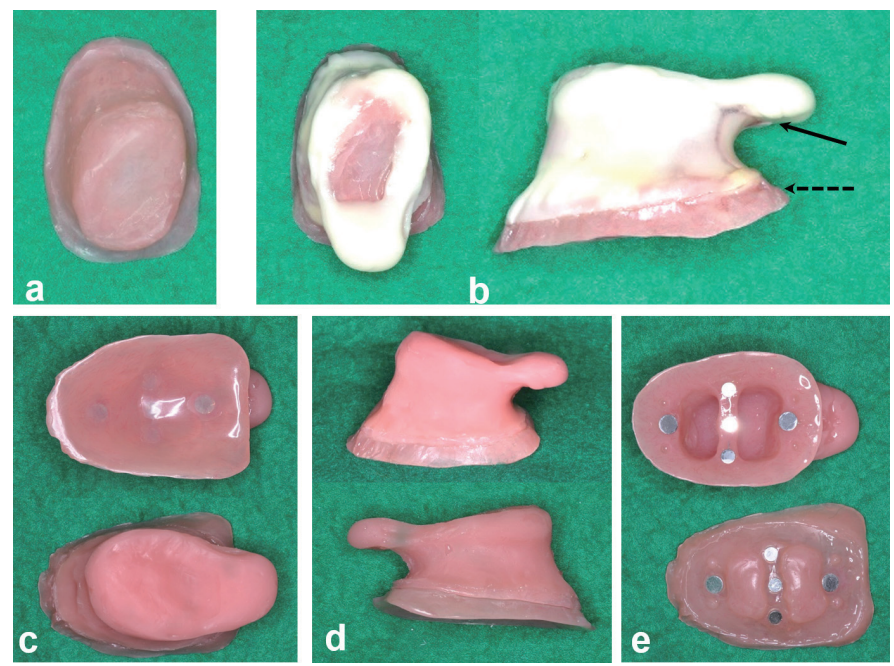

Fig. 3 The retainer-free obturator prosthesis, without undercuts (a). The obturator was extended to the undercut on the nasopharyngeal side of the soft palate with self-cured resin and tissue conditioner (b). The fabricated retainer-free obturator prosthesis: occlusal and mucosal views (c), lateral views (d), and separated sectional obturator prosthesis (e)

istics (e.g., swallowing, speaking, obturator retention) had improved, the tissue conditioner on the obturator was replaced with soft relining material (GC Reline Extra Soft; GC Corp.). Figure 3 c-e shows the completed retainer-free obturator prosthesis. The prosthesis consisted of a palatal plate and hollow obturator portion with a resilient silicone layer over a resin frame, to engage the deep undercuts within the defect and help retain the prosthesis. The clasp-retained obturator prosthesis weighed $19 \mathrm{~g}$; in contrast, the retainer-free obturator prosthesis weighed $14 \mathrm{~g}$. The length and width of the palatal plate were 4.5 and $3.3 \mathrm{~cm}$, respectively, and the length, width, and height of the obturator portion were $5.2,2.5$, and 1.8 $\mathrm{cm}$, respectively. Figure 4 shows intraoral views with (Fig. 4a) and without (Fig. 4b) the obturator prosthesis (June 2019). The patient was instructed
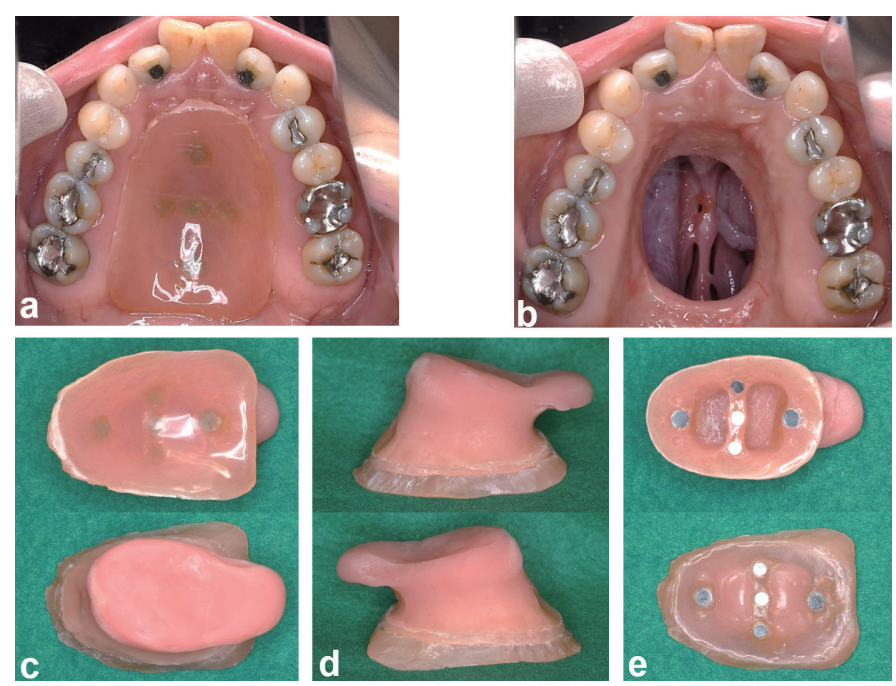

Fig. 4 Intraoral view with (a) and without (b) the retainer-free obturator prosthesis. Occlusal and mucosal views (c), lateral views (d), and the separated sectional obturator prosthesis (e) at 5 years after insertion

to insert and remove the obturator carefully to prevent damage to surrounding tissues. To be precise, she was instructed to insert the retainer-free obturator prosthesis from a posterior tilt and push on the palatal plate to rotate it into position. She was also directed to put her fingers on the front end of the palate plate and rotate it to remove the obturator prosthesis. In addition, she was instructed to clean its inner surface after separating the obturator into two sections. Although her oral hygiene has generally been good, continuing instruction regarding oral hygiene, including tooth brushing and cleaning of the prosthesis, is provided at every periodic follow-up examination. Follow-up examinations are conducted every 3 months, and no clinical problems were observed at the latest (5-year) follow-up.

Table 1 shows the results for the retainer-free obturator prosthesis, 
namely, masticatory performance, blowing ratio, visual analog scale, and patient mood assessment (June 2019). All measurements were performed by a single dentist who was blinded to the type of obturator prosthesis used by the patient. The results of the evaluations (especially those for the chewing assessment and esthetics) were much better for the retainer-free obturator prosthesis than for the clasp-retained obturator prosthesis.

\section{Discussion}

A retainer-free obturator prosthesis was used to treat hard and soft palatal defects in a fully dentulous patient. The use of a sectional obturator prosthesis that engages the deep undercuts, thus interfering with insertion/ removal, has been reported in partially edentulous maxillectomy patients [6]. However, this appears to be the first report of a case in which a retainer-free obturator prosthesis was inserted in a fully dentulous patient after tumor resection.

The open, hollow obturator is difficult to polish and clean, which leads to accumulation of food and debris inside the hollow portion [7]. In contrast, the closed, hollow obturator has a sealed area, which is a potential site of leakage. To compensate for these disadvantages, a removable, closed, hollow obturator with an attachment was fabricated. The obturator and palatal plate can thus be separated, allowing the internal aspect of the obturator to be easily cleaned by the patient. Magnetic attachment was selected on the basis of the thickness of the part where the attachment is placed. The use of multiple magnets, however, is somewhat more expensive than other attachment methods, and the time required for fabrication is longer than that needed for the conventional technique. The number and location of the magnetic attachments were determined by trial and error. A trial placement of the retainer-free obturator prosthesis was performed. Initially, two magnetic attachments (Gigauss D800) were placed at the front and rear positions. However, the obturator rotates at the attachment and detachment, and a satisfactory retentive force between the palatal plate and obturator portion could not be obtained. To prevent accidental ingestion of the prosthesis, the number of magnetic attachments was increased (Gigauss D400). The retentive force of the magnetic attachment and obturator should be monitored regularly.

To reduce prosthesis weight and prevent deformation, the obturator portion was composed of a thin silicone layer lining an acrylic resin frame. A potential alternative to exploiting the retentive parts of the defect is the use of a semi-flexible thermoplastic resin, such as polyamide, polyester, or polypropylene. Because of its durability, a silicone-type, soft relining material was selected. Wright reported that a heat-cured, silicone-type, soft relining material (Molloplast-B) continued to function after more than 5 years of use [8]. The present report used an auto-polymerized, siliconetype, soft relining material, which is covered by the health insurance system in Japan. Because the long-term clinical prognosis of this material is unclear, it is important to conduct periodic checkups (at shorter intervals than those for conventional dentures) and replace the material, if necessary. Figure $4 \mathrm{c}$-e shows the obturator prosthesis at 5 years after insertion. The soft relining material of the retainer-free obturator prosthesis has not yet been replaced. Severe discoloration and peeling — which are problems with long-term use of soft relining material [9] — were not observed. This relining material will likely be stable for a long time, because occlusal force does not act directly on the obturator, and saliva has little direct effect.

Within the limitations of the 5 years of follow-up in this case, the present findings suggest that a newly designed retainer-free obturator prosthesis provides satisfactory esthetics and addresses the problems of food residue accumulation and nasal drainage caused by the use of retainers in fully dentulous patients.

\section{Acknowledgments}

The authors thank Yoshihisa Takenouchi, prosthetic laboratory technician at A-Dental Laboratory, for his assistance. This work was supported in part by a JSPS KAKENHI Grant (17K12052).

\section{Conflict of interest}

None declared.

\section{References}

1. Keyf F (2001) Obturator prostheses for hemimaxillectomy patients. J Oral Rehabil 28 , 821-829.

2. Murakami M, Nishi Y, Umezono M, Kamashita Y, Nishimura M (2015) Fabrication of a movable obturator following maxillary reconstruction with slit-shaped fenestration. J Prosthodont 24, 254-259.

3. Aramany MA (1978) Basic principle of obturator design for partially edentulous patients. Part 2: design principle. J Prosthet Dent 40, 656-662.

4. Aramany MA (1978) Basic principles of obturator design for partially edentulous patients. Part 1: classification. J Prosthet Dent 40, 554-557.

5. Katoh C, Saitoh M, Tsuneyuki M, Tanimoto H, Hashikawa K, Tahara S et al. (2010) Blowing ratio as an evaluation tool for velopharyngeal function after oral and oropharyngeal cancer resection. Head Neck 32, 1012-1018.

6. Matsumura H, Kawasaki K (2000) Magnetically connected removable sectional denture for a maxillary defect with severe undercut: a clinical report. J Prosthet Dent 84, 22-26.

7. Yazicioğlu H, Yaluğ S (2002) An alternative method for fabricating a closed hollow obturator: a clinical report. J Oral Sci 44,161-164.

8. Wright PS (1994) Observations on long-term use of a soft-lining material for mandibular complete dentures. J Prosthet Dent 72, 385-392.

9. Tanimoto Y, Saeki H, Kimoto S, Nishiwaki T, Nishiyama N (2009) Evaluation of adhesive properties of three resilient denture liners by the modified peel test method. Acta Biomater $5,764-769$. 